\title{
0 papel da imagem corporal positiva na qualidade de vida de pessoas com insuficiência renal crónica
}

\author{
Margarida S. Cunha (iD) 1, Filipa E. Vieira ${ }^{1,2}$, Rita S. Cepeda 3 , Sandra C. Torres ${ }^{1,2}$, \& Maria \\ Raquel Barbosa ${ }^{1,2}$ \\ ${ }^{1}$ Faculdade de Psicologia e Ciências da Educação da Universidade do Porto (FPCEUP) \\ ${ }^{2}$ Centro de Psicologia da Universidade do Porto \\ ${ }^{3}$ Clínica Tecsam - Tecnologia e Serviços Médicos, S.A., Mirandela, Vila Real, Portugal
}

\begin{abstract}
Resumo: Este estudo pretende explorar o papel da imagem corporal positiva (ICP) na qualidade de vida (QdV) de pessoas com insuficiência renal crónica (IRC). A recolha de dados foi efetuada através de instrumentos de autorrelato que permitiram a contemplação das variáveis sociodemográficas, de algumas dimensões da ICP e da QdV, num grupo clínico ( $\mathrm{n}=46 ; M=59,8$ anos) e num grupo não clínico ( $\mathrm{n}=49 ; M$ = 55,1 anos) de participantes. Percebeu-se que as várias dimensões da ICP se associam com os diferentes domínios da QdV em ambos os grupos. As mulheres revelaram níveis superiores nas dimensões da aceitação do corpo pelos outros e no autocuidado, comparativamente aos homens. Por último, destaca-se o papel da apreciação da funcionalidade corporal como um importante preditor da QdV. Salienta-se a relevância de se intervir na ICP pelo impacto positivo que pode ter na QdV desta população.
\end{abstract}

Palavras-chave: Imagem corporal positiva; Insuficiência renal crónica; Hemodiálise; Qualidade de vida.

The role played by positive body image on the quality of life of patients with chronic kidney disease: This study aims to explore the role of positive body image (PBI) in the quality of life (QoL) of people with chronic renal failure (CRF). Data collection was performed using self-report instruments that allowed sociodemographic variables, some dimensions of PBI and QoL, in a clinical group ( $\mathrm{n}=46 ; M=59.8$ years) and in a non-clinical group ( $\mathrm{n}=49 ; M=55.1$ years) of participants. It was founded a positive association between PBI dimensions and the different domains of QoL in both groups. Women showed higher levels in the dimensions of body acceptance by others and self-care, compared to men. Finally, the results underlined the role of body functionality appreciation has as an important predictor of QoL. The importance of intervening in PCI is highlighted due to the positive impact it can have on the QoL of this population.

Keywords: Positive body image; Chronic Kidney Disease; Hemodialysis; Quality of life.

A insuficiência renal crónica (IRC) é uma diminuição lenta e progressiva (durante meses ou anos) da capacidade dos rins de filtrar os resíduos metabólicos do sangue. Os efeitos da IRC na pessoa podem ser nefastos, em situações mais graves e avançadas da doença, e manifestam-se a diversos níveis, nomeadamente, a nível biológico, psicológico e em termos de imagem corporal (IC), uma vez que a IRC tem implicações corporais, independentemente do tratamento selecionado (e.g., formação de cicatrizes, alterações musculoesqueléticas, alterações oculares) (Liew et al., 2012).

\begin{abstract}
A Imagem Corporal em Pessoas com Insuficiência Renal Crónica
"A IC engloba elementos como a perceção do tamanho do corpo (perceções), avaliação da atratividade do corpo (pensamentos) e emoções associadas com a forma e o tamanho do corpo (sentimentos)" (Grogan, 1999, p. 1). Contudo, quando se fala de IC é preciso olhar para o conceito do ponto de vista psicológico e sociológico, uma vez que a IC é um fenómeno psicológico que é significativamente afetado por fatores sociais. Portanto, para se poder compreender o fenómeno na totalidade, é necessário olhar não apenas para as experiências dos indivíduos, mas também para o meio cultural em que o indivíduo se situa (Grogan, 1999).

Assim, a IC é aqui concetualizada como subjetiva e aberta à mudança por influência social, não existindo elo necessário entre a experiência subjetiva do corpo de uma pessoa e o que é percebido por um

${ }^{1}$ Morada para correspondência: Maria Raquel Barbosa, Faculdade de Psicologia e Ciências da Educação da Universidade do Porto, Rua Alfredo Allen, s/n, 4200-135, Porto, Portugal. Email: raquel@fpce.up.pt
\end{abstract}


observador externo. Isso é óbvio na distorção do tamanho corporal (por exemplo, muitas mulheres jovens que experimentam anorexia nervosa acreditam que são muito mais pesadas do que realmente são) (Grogan, 1999).

A vivência da IC é de suma importância na forma como o indivíduo lida com a doença. A investigação (e.g., Finnegan-John \& Thomas, 2012; Partridge \& Robertson, 2011) tem revelado que pessoas em processo de hemodiálise apresentam níveis mais elevados de perturbação da IC e níveis mais reduzidos de qualidade de vida $(Q d V)$. A melhoria da IC, por sua vez, influencia significativamente na recuperação psicossocial, na adaptação à doença e na QdV (Partridge \& Robertson, 2011).

O conceito de imagem corporal positiva (ICP), por seu lado, é um construto inovador e peculiar, que não está no continuum do construto de imagem corporal negativa nem deve ser representada por baixos níveis de imagem corporal (Tylka, 2011). Neste seguimento, é essencial salientar que a ICP não se caracteriza pela ausência de imagem corporal negativa e, mais do que a satisfação com o corpo, abrange outras variáveis que tornam este conceito multidimensional e holístico (Wood-Barcalow et al., 2010).

Assim, como referido previamente, a ICP pode ser melhor explicada através de várias características como; a) o seu caráter holístico, na medida em que as múltiplas facetas da ICP são mais bem interpretadas juntas do que de forma independente (Wood-Barcalow et al., 2010); b) a sua estabilidade e maleabilidade, pois integra características de estado e de traço da personalidade (Tylka \& Wood-Barcalow, 2015a); c) e o seu caráter complexo e multifacetado, fazendo-se constituir por dimensões como aceitação corporal e amor, apreciação do corpo, concetualização ampla de beleza, perceção da aceitação do corpo pelos outros, apreciação e aceitação da funcionalidade corporal e o autocuidado (Tylka \& Wood-Barcalow, 2015a).

Apesar deste seu caráter multifacetado, a ICP é comummente operacionalizada pelo conceito de apreciação corporal, que envolve elogiar o corpo pelo que ele é capaz de fazer, o que representa e as suas características singulares (Bailey et al., 2016; Tylka \& Wood-Barcalow, 2015a). Esta atitude implica expressar conforto em relação à aparência, mesmo não se estando completamente satisfeito com todos os seus aspetos, podendo experimentar perceções, sentimentos, pensamentos e sensações aversivas em relação ao seu corpo, sem tentativas de suprimir ou desviar essas experiências (Bailey et al., 2016). Além disso, permite a proteção face a uma IC irrealista, uma vez que existe a capacidade de filtrar informações de forma protetora (Wood-Barcalow et al., 2010).

Contudo, devido ao supracitado, decidimos ainda incluir, neste estudo, outras dimensões da ICP como a apreciação da funcionalidade do corpo (definida como tudo o que o corpo pode fazer ou é capaz de fazer, valorizando-o, respeitando-o e honrando-o por isso; Alleva et al, 2017); a aceitação do corpo pelos outros (definida pela perceção que o próprio tem acerca das avaliações que os outros fazem do seu corpo; Avalos \& Tylka, 2006; Wood-Barcalow et al., 2010) e o autocuidado (definido pelo investimento no cuidado com o corpo e nas suas necessidades físicas; Tylka, 2011).

Portanto, apesar da insatisfação com a IC ser uma consequência psicológica comum de viver com IRC (Finnegan-John \& Thomas, 2012), é possível ter uma ICP, uma vez que esta não simboliza ter um corpo perfeito alinhado com o ideal cultural (Tylka \& Wood Barcalow, 2015a), mas antes a aceitação do corpo, a concentração nos seus aspetos positivos, a apreciação e gratidão corporal (Bailey et al., 2015).

Paralelamente, torna-se relevante realçar a relação que a ICP estabelece com a variável sexo e idade. A investigação tem sido inconclusiva acerca de que forma a apreciação do corpo difere em função do sexo. Alguns autores não encontraram diferenças estatisticamente significativas entre homens e mulheres na imagem corporal positiva, tanto em estudos internacionais (e.g., Razmus \& Razmus, 2017) como nacionais (e.g., Meneses et al., 2019). Por sua vez, Atari (2016) ou Swami e Ng (2015) encontraram níveis superiores de apreciação corporal no sexo masculino, mas Jovic et al., (2016), por seu lado, referem níveis superiores no sexo feminino.

Relativamente às diferenças de idade na ICP, a evidência sugere que, com a idade, os indivíduos parecem apreciar mais a sua saúde e funcionalidade e são mais capazes de aceitar as imperfeições corporais, ao mesmo tempo que atribuem menos importância à sua aparência física (Tiggemann, 2004).

\section{A Imagem Corporal e a Qualidade de Vida}

O estudo da QdV e da sua relação com a ICP revela-se importante não só pelo facto do estudo da IC se ter centrado essencialmente no seu aspeto negativo e nos seus desfechos adversos (Moreira \& Canavarro, 2010), nomeadamente na insatisfação corporal, como pelo facto de existir escassa investigação neste âmbito. A investigação tem revelado uma relação importante entre a IC e a QdV, tanto em pessoas com IRC (Finnegan-John \& Thomas, 2012; Partridge \& Robertson, 2011), como em pessoas sem IRC (Moreira \& Canavarro, 2010), especificamente que níveis mais elevados de insatisfação corporal estão associados a 
uma menor QdV. Todavia, não foram encontrados estudos que explorassem a relação existente entre a ICP e a QdV na presente população clínica² .

\section{Objetivos e Hipóteses}

Uma vez que a investigação nesta área é escassa, o presente trabalho pretende ser um contributo para um maior conhecimento sobre o papel da ICP na QdV de pessoas com IRC, em processo de hemodiálise. Tendo em consideração a revisão bibliográfica acerca do tema em questão, foram elaboradas as seguintes hipóteses de trabalho:

H1. Espera-se que as dimensões da ICP apresentem valores estatística e significativamente mais elevados no grupo não clínico (GNC) que no grupo clínico (GC) (Finnegan-John \& Thomas, 2012; Liew et al., 2012; Partridge \& Robertson, 2011; Richard \& Engebretson, 2010).

H2. Não são esperadas diferenças de sexo nas diferentes dimensões da ICP, independentemente do grupo (Meneses et al., 2019; Razmus \& Razmus, 2017), à exceção do autocuidado que se espera estatística e significativamente inferior no sexo masculino (Pengpid \& Peltzer, 2015).

H3. Espera-se que as dimensões da ICP (a apreciação da funcionalidade corporal, a apreciação corporal, a aceitação do corpo pelos outros e o autocuidado) se correlacionem de forma positiva e estatisticamente significativa entre si (Alleva et al., 2017; Avalos \& Tylka, 2006; Bailey et al., 2015; Tylka \& Wood-Barcalow, 2015a; Wood-Barcalow et al., 2010) e com a QdV (Finnegan-John \& Thomas, 2012; Moreira \& Canavarro, 2010; Partridge \& Robertson, 2011), em ambos os grupos.

H3.1. Espera-se, também, uma associação positiva entre a idade e a ICP (Atari, 2016; Swami \& Ng, 2015; Tiggemann, 2004) e entre a idade e a QdV (Oldenburg et al., 2013), em ambos os grupos.

H4. Espera-se que as dimensões da ICP (BAS-2, FAS, BAOS e BRS), o grupo e a idade sejam preditores positivos e significativos da QdV (Moreira \& Canavarro, 2010).

\section{MÉTODO}

\section{Desenho Metodológico}

O presente estudo recorre a uma metodologia quantitativa, com um desenho de investigação observacional, analítico e transversal, com GC e GNC.

\section{Participantes}

Os critérios de inclusão dos participantes no GC foram os seguintes: a apresentação de diagnóstico médico de IRC e prática de hemodiálise há, pelo menos, 1 ano; acuidade visual normal (ou corrigida para normal), bem como a presença de uma idade igual ou superior a 18 anos. Os critérios de exclusão foram: apresentação de algum défice cognitivo ${ }^{3}$; gravidez ou um período de 6 meses pós-gravidez; patologia oftalmológica significativa; disforia de género; comportamentos de automutilação; comorbilidade psiquiátrica severa, bem como exclusão de todos os participantes que se encontravam em estado crítico ou em cuidados paliativos.

Quanto ao grupo controlo ou não clínico os critérios de inclusão foram: idade cronológica igual ou superior a 18 anos; peso normal (isto é, um índice de massa corporal entre os 18.5 e $25 \mathrm{~kg} / \mathrm{m} 2$ ) e um período menstrual regular (em mulheres pré-menopausa). Por seu lado, foram restritos de participar todos os participantes com uma história atual ou passada de doença médica ou psiquiátrica severa.

O GC é constituído por 46 participantes, com uma representatividade equitativa quanto ao sexo (50\% homens e 50\% mulheres), com uma média de idade de 59.8 anos $(D P=14.5)$, variando entre os 30 e os 85 anos de idade. A média de anos de escolaridade é de 8.82 anos $(D P=4.66)$, variando entre a ausência de escolaridade até aos 21 anos de ensino. 58.7\% dos participantes encontra-se casado ou em união de facto, $19.6 \%$ dos participantes é solteiro, $15.2 \%$ são viúvos e $6.5 \%$ encontram-se divorciados/separados. Os participantes com IRC apresentam a doença, em média, há 137 meses $(D P=131)$.

O GNC é constituído por 49 participantes, $49 \%$ dos quais são do sexo masculino e $51 \%$ são do sexo feminino. Quanto à idade, os participantes apresentam em média 55.1 anos $(D P=10.0)$, variando entre os 32 e os 75 anos de idade. A média de escolaridade é de 13.1 anos $(D P=4.51)$, variando entre os 4 e os 27 anos de escolaridade. $83.7 \%$ dos participantes encontra-se casado ou em união de facto, $6.1 \%$ dos participantes é solteiro, $6.1 \%$ encontram-se divorciados/separados e $4.1 \%$ são viúvos.

\footnotetext{
${ }^{2}$ De referir que a exploração se baseou nas bases de dados Scopus, Web of science, PsycInfo e Medline, utilizando as seguintes combinações de palavras-chave: chronic kidney disease OR chronic renal failure OR hemodialysis AND Quality of life AND Positive body image OR Body appreciation. Foram explorados estudos realizados com amostras de pacientes adultos e idosos em hemodiálise e sem limite de data de publicação.

${ }^{3}$ Este critério foi validado pelo elo de ligação na clínica onde foi realizada a recolha. Na recolha online, o facto de o método se basear numa amostragem não probabilística por bola de neve, permitiu algum controlo face a esta variável.
} 
De realçar que foi realizado um emparelhamento prévio entre os participantes do GC e do GNC em função do sexo e idade, de modo a assegurar a homogeneidade entre grupos em relação às presentes variáveis sociodemográficas. Contudo, esta equivalência não foi conseguida em termos de escolaridade, com o GNC mais escolarizado $(M=13.1, D P=4.51)$, comparativamente ao GC $(M=8.82, D P=4.66), t(91)$ $=-4.33 ; p \leq .001 ; d=-.93$. Tal aspeto será tido em conta em análises posteriores ${ }^{4}$ (c.f. Tabela 1 ).

Tabela 1. Caracterização dos participantes e comparação entre GC e GNC quanto às variáveis sociodemográficas

\begin{tabular}{|c|c|c|c|c|c|c|}
\hline & \multirow{2}{*}{$\begin{array}{c}\text { GC } \\
(n=46)\end{array}$} & \multirow{2}{*}{$\begin{array}{c}\text { GNC } \\
(n=49)\end{array}$} & \multicolumn{4}{|c|}{ Comparação entre grupos } \\
\hline & & & $t / \chi^{2}$ & $g l$ & $p$ & $d / \varphi$ \\
\hline \multicolumn{7}{|l|}{ Sexo n (\%) } \\
\hline Masculino & $23(50 \%)$ & $24(49 \%)$ & & & & \\
\hline Feminino & $23(50 \%)$ & $25(51 \%)$ & & & & \\
\hline Escolaridade $\mathrm{M} \pm \mathrm{DP}$ & $8.82 \pm 4.66(n=44)$ & $13.14 \pm 4.51$ & -4.54 & 91 & .000 & -.93 \\
\hline Mínimo - Máximo & $0-21$ & $4-27$ & & & & \\
\hline Idade $M \pm D P$ & $59.78 \pm 14.50$ & $55.12 \pm 10.00$ & & & & \\
\hline Mínimo - Máximo & $30-85$ & $32.0-75.0$ & & & & \\
\hline Estado Civil n (\%) & & & 2.05 & 3 & .56 & .15 \\
\hline Solteiro(a) & $9(19.6 \%)$ & $3(6.1 \%)$ & & & & \\
\hline Casado(a) & $27(58.7 \%)$ & $41(83.7 \%)$ & & & & \\
\hline Divorciado(a) & $3(6.5 \%)$ & $3(6.1 \%)$ & & & & \\
\hline Viúvo(a) & $7(15.2 \%)$ & $2(4.1 \%)$ & & & & \\
\hline Doença $M \pm D P$ (meses) & $137 \pm 131$ & ------ & & & & \\
\hline
\end{tabular}

Nota. GC = Grupo Clínico; GNC = Grupo Não Clínico.

\section{Instrumentos}

A recolha de dados foi efetuada através de vários instrumentos de autorrelato que permitiram a contemplação de variáveis sociodemográficas, de algumas dimensões da ICP (apreciação corporal, funcionalidade corporal, aceitação do corpo pelos outros e autocuidado) e da QdV.

\section{Variáveis Sociodemográficas}

Questionário Sociodemográfico. Foi administrado um questionário de registo de informações sociodemográficas e clínicas relevantes para a investigação, desenvolvido para esse efeito. Desta forma, conseguiu-se recolher informação acerca de cada participante, nomeadamente, idade, sexo, escolaridade, profissão e estado civil, diagnóstico e tratamento, dados antropométricos, grau de satisfação com a imagem corporal e grau de positividade. Por último, colocaram-se questões específicas para a condição clínica da insuficiência renal crónica, nomeadamente, tipo e regime de tratamento, número de horas semanais despendidas com o tratamento, candidato a transplante renal e alterações corporais consequentes da doença/tratamento.

\section{Imagem Corporal Positiva}

Apreciação Corporal. A apreciação corporal foi avaliada pela Body Appreciation Scale-2 (BAS- 2, de Tylka \& Wood-Barcalow, 2015b; versão portuguesa de Lemoine et al., 2018). A BAS-2 avalia a aceitação, as opiniões favoráveis e o respeito que os indivíduos têm pelo seu próprio corpo. A escala é constituída por 10 itens, respondidos numa escala tipo likert de 1 (nunca) a 5 (sempre) (níveis mais elevados revelam uma melhor apreciação pelo corpo, isto é, uma ICP mais elevada, Tylka \& Wood-Barcalow, 2015b). A escala original apresenta bons índices de fiabilidade $(\alpha=.97)$ e os resultados dos estudos em amostras portuguesas suportam, também, a sua fidelidade, tanto em adultos mais jovens $(\alpha=.94$; Lemoine et al., 2018) como em adultos mais velhos ( $\alpha=.88$; Meneses et al., 2019). Nas amostras deste estudo o valor de consistência interna foi de $\alpha=.91$ para o GC e de $\alpha=.90$ para o GNC. A amostra total teve um $\alpha=.90$.

\footnotetext{
${ }^{4}$ Foram realizados testes qui-quadrado para a independência das variáveis ou testes t para amostras independentes e, depois de cumprido o pressuposto de igualdade das variâncias para todas as variáveis métricas, não foram encontradas diferenças estatisticamente significativas entre os grupos quanto ao estado civil e IMC. Contudo, não foi possível a igualdade quanto à variável escolaridade. De referir que a variável escolaridade não se refere ao nível de escolarização, mas sim ao número de anos que os participantes estudaram.
} 
Funcionalidade. Avaliada pela Functionality Appreciation Scale (FAS, de Alleva et al., 2017). Esta medida tem como objetivo avaliar a apreciação da funcionalidade do corpo e a valorização, respeito e honra do corpo em função do que este é capaz de fazer, tentando ir para além de uma simples consciência da funcionalidade corporal. A FAS apresenta uma estrutura unidimensional e é constituída por 7 itens, respondidos numa escala tipo likert de 1 (discordo totalmente) a 5 (concordo totalmente). A cotação da escala efetua-se através da média das pontuações dos participantes nos itens. Pontuações mais altas refletem uma maior apreciação da funcionalidade. A FAS apresenta uma boa consistência interna $(\alpha=.91)$ e estabilidade temporal com três semanas de intervalo (Alleva et al., 2017). É uma escala ainda não validada para a população portuguesa, sendo o presente estudo parte integrante desse processo. Neste trabalho os alfas foram: $\alpha=.91$ para o GC, $\alpha=.86$ para o GNC e um $\alpha=.90$ na amostra total.

Aceitação Corporal Pelos Outros. Avaliada pela Body Acceptance by Others Scale (BAOS, de Avalos \& Tylka, 2006; versão portuguesa de Barbosa et al., 2018). Avalia a aceitação percebida do próprio corpo pelos outros (família, amigos, namorados e media). A escala é constituída por 10 itens, respondidos numa escala tipo likert de 1 (nunca) a 5 (sempre). Avalos e Tylka (2006) demonstraram a presença de uma boa consistência interna $(\alpha=.91)$, fidelidade teste-reteste, validade de construto (convergente e discriminante) e incremental deste instrumento. Os valores de consistência interna da versão portuguesa da escala têm demonstrado níveis satisfatórios, tanto em amostras de adolescentes $(\alpha=.93)$ e adultos $(\alpha=.94$; Barbosa et al., 2018) como em idosos ( $\alpha=.94$; Meneses et al., 2019). Neste estudo os índices de consistência interna foram de o $\alpha=.89$ no GC, $\alpha=.94$ no GNC e $\alpha=.92$ na amostra total.

Autocuidado. 0 autocuidado foi avaliado através da Body Responsiveness Scale (BRS, de Daubenmier, 2005). A escala é constituída por 7 itens, devendo ser respondidos numa escala tipo likert de 1 (nada verdadeiro a meu respeito) a 5 (muito verdadeiro a meu respeito). A autora original da escala refere uma estrutura unidimensional, com boa consistência interna, $(\alpha=.83$, Daubenmier, 2005).

Esta é, também, uma escala ainda não validada para a população portuguesa, sendo o presente estudo parte integrante desse processo. Nas amostras deste estudo foi obtido um $\alpha=.53$ no GC, $\alpha=.68$ no GNC e $\alpha=.61$ na amostra total.

\section{Qualidade de Vida}

A QdV foi avaliada através de um instrumento desenvolvido em colaboração com vários centros e em diversos contextos culturais ao longo de vários anos, pelo grupo WHOQOL (World Health Organization Quality Of Life [WHOQOL], 1998), em específico, pelo WHOQOL-BREF, uma versão abreviada do WHOQOL100. O WHOQOL-BREF fornece uma alternativa válida e confiável ao WHOQOL-100, acabando por ser mais útil em estudos que exigem uma breve avaliação da QdV e para profissionais de saúde na avaliação da eficácia do tratamento, uma vez que é de fácil aplicação e o preenchimento é menos moroso. A escala é constituída por 26 itens, organizados em 4 domínios relacionados com a QdV: física, psicológica, relacional e ambiental. Também inclui uma faceta da QdV e da saúde em geral. Apresenta validade de construto discriminante, validade de conteúdo, boa consistência interna (entre $\alpha=.66$ no domínio QdV relacional e $\alpha$ $=.84$ no domínio QdV física) e fidelidade teste-reteste. Neste estudo foi utilizada a versão portuguesa da WHOQOL-BREF de Serra e colaboradores (2006). 0 alpha de Cronbach deste instrumento varia entre 64 (QdV relacional) e .87 (QdV física), sendo que os domínios em conjunto explicam 58.8\% da variância (Serra et al., 2006). Neste estudo o alpha de Cronbach varia entre .63 ( $Q \mathrm{dV}$ geral) e .87 ( $\mathrm{dV}$ física), na amostra total, entre .62 (QdV relacional) e .84 (QdV física) no GC e entre .20 (QdV geral) e .82 (QdV ambiental) no $\mathrm{GNC}^{5}$.

\section{Procedimento}

Relativamente à recolha da amostra, as amostras foram recolhidas com base num processo de amostragem não probabilístico, por conveniência ou pelo método de bola de neve.

A recolha presencial da amostra clínica teve lugar numa clínica do Norte, que acedeu participar no estudo, após o estabelecimento de um contacto via email. Os questionários foram administrados em papel, por dificuldades de acesso à internet neste local. Paralelamente, a recolha de dados foi realizada durante as sessões de hemodiálise e, portanto, em forma de entrevista, devido à posição em que os participantes se encontravam - deitados e, muitas vezes, com a fístula na mão que escreve. Virtualmente, recorreu-se à Associação Portuguesa de Insuficientes Renais (APIR), a alguns contactos sociais e a um grupo de IRC nas redes sociais.

\footnotetext{
${ }^{5}$ Embora se considerem aceitáveis $\alpha>.60$, considerando-se com uma confiabilidade moderada algumas das medidas de QdV, devido ao valor muito baixo de confiabilidade da subescala de QdV geral no GNC, optou-se por não incluir esta medida nas análises posteriores. Os resultados da BRS devem ser analisados com cautela.
} 
Quando contactada a instituição para realizar a recolha, a mesma elegeu um elo de ligação, disponível para agilizar o processo. Assim, os participantes do GC foram convidados a participar no estudo pelo seu próprio médico ou psicólogo (elo de ligação), após a ponderação dos critérios de inclusão e exclusão definidos. Todos os participantes foram informados sobre: (a) os objetivos do estudo; (b) a ausência de riscos expectáveis na participação; (c) os procedimentos que compõem o estudo (i.e., preenchimento de medidas de autorrelato e realização de uma entrevista), e (d) o tempo necessário para a participação (aproximadamente 30 minutos, conforme o teste piloto efetuado). Todas as informações fornecidas oralmente pela investigadora foram também colocadas por escrito numa Folha de Informação ao Participante entregue ao mesmo. Foi reforçado que a sua participação é voluntária e confidencial. Antes da recolha de dados ter início, foi preenchido, também, o Consentimento Informado.

A amostra do GNC foi recolhida online, através da plataforma da UP limesurvey. Os participantes foram contactados pessoalmente, via e-mail ou redes sociais, com o objetivo de apresentar o estudo e o interesse em recolher amostra. As pessoas cuja resposta foi positiva preencheram o questionário com acesso através da partilha de um link. Todos os participantes, depois de lerem as informações acerca do estudo e seus objetivos, tiveram, igualmente, que dar o seu consentimento informado, sem o qual o preenchimento do questionário não era possível.

O protocolo é composto por 14 páginas, ordenadas de forma contrabalançada, e o seu preenchimento durou, em média, 90 minutos, para o GC e 30 minutos para o grupo não-clínico. 0 período de coleta realizou-se entre o dia 17 de dezembro de 2019 e 24 de abril de 2020.

Relativamente aos procedimentos éticos e de proteção de dados, este projeto respeita os princípios éticos enunciados na Declaração de Helsínquia, considerando a participação voluntária, o consentimento informado e esclarecido, a confidencialidade e o acesso aos dados.

Todos os instrumentos utilizados tiveram a autorização dos autores originais ou dos autores das versões nacionais.

0 estudo teve o parecer positivo da Comissão de Ética Institucional (Parecer n. $2028 / 12-6 b$ ) e da Avaliação de Impacto da Proteção de Dados da UP (AIP-UP; Ref. 4/2020).

Em termos de procedimentos estatísticos, após a administração da bateria de instrumentos, procedeu-se à codificação dos dados e posterior tratamento estatístico, com recurso ao programa de análise estatístico IBM SPSS Statistics (Statistical Package for the Social Sciences), na versão 26.

Foi realizada a limpeza da base de dados e foram avaliadas as eventuais violações de pressupostos estatísticos que desvirtuassem os resultados. No total foram eliminados 7 participantes (4 do GC e 3 do GNC), por não cumprirem os critérios de inclusão (e.g., já não realizavam hemodiálise) ou por terem mais de $25 \%$ de missing values.

Foram realizadas análises descritivas para caracterizar a amostra e as variáveis em estudo; testes $t$ student para amostras independentes e testes qui-quadrado para a independência das variáveis, para avaliar a equivalência dos grupos nas diferentes variáveis sociodemográficas; two-way ANCOVAS para comparar os resultados dos dois grupos nas diferentes variáveis dependentes, controlando o efeito da escolaridade; testes de correlação usando o coeficiente de Pearson para averiguar as associações das variáveis em estudo no GC e não clínico e, ainda, um modelo de regressão linear múltipla, para se explorarem os principais preditores da qualidade de vida.

O pressuposto da normalidade da distribuição foi explorado e confirmado recorrendo aos testes de Kolmogorov-Smirnorv, bem como aos valores de assimetria e curtose da distribuição, concluindo-se que todas as variáveis apresentam uma distribuição normal $(|s k|<2$; $|k u|<7$; Kline, 2011). Relativamente às ANCOVAS e à análise de Regressão Linear Múltipla, foram analisados e cumpridos os pressupostos que viabilizam a prossecução das análises.

\section{RESULTADOS}

\section{Diferenças de Sexo e de Grupo nas Dimensões da Imagem Corporal Positiva}

Para a avaliação de eventuais efeitos do grupo (clínico e não clínico) e do sexo (feminino e masculino), relativamente às variáveis dependentes em estudo, foram realizadas diversas two-way ANCOVAS, controlando-se o efeito da escolaridade (covariável).

Não foram encontrados efeitos de interação entre sexo e grupo estatisticamente significativos na apreciação corporal, $F(1,88)=1.79, p=.184, \eta^{2} p=.02$, na aceitação do corpo pelos outros, $F(1,88)=1.55$, $p=.217, \eta^{2} p=.02$, na apreciação da funcionalidade, $F(1,88)=0.45, p=.503, \eta^{2} p=.01$, e no autocuidado, $F$ $(1,88)=0.17, p=.678, \eta^{2} p=.00$, enquanto se controlava o efeito da escolaridade.

Contudo, verificou-se um efeito principal do grupo e dois efeitos principais do sexo. Assim, assinalase uma diferença estatisticamente significativa na apreciação da funcionalidade corporal entre os participantes do GC e do GNC, $F(1,88)=5.44, p=.022, \eta^{2} p=.06$, com os participantes do GNC a 
apresentarem uma apreciação da funcionalidade corporal mais positiva que os participantes do GC $(M=$ $4.3, D P=0.1, n=49$ e $M=3.9, D P=0.1, n=44$, respetivamente).

Relativamente ao efeito principal do sexo, e controlando o efeito da escolaridade, constatou-se uma diferença estatisticamente significativa na avaliação percecionada da aceitação corporal por parte dos outros entre os participantes do sexo masculino e do sexo feminino, $F(1,88)=9.19, p=.003, \eta^{2} p=.10$. Isto é, em média, os sujeitos do sexo feminino percecionam níveis mais elevados de aceitação corporal por parte dos outros do que os participantes do sexo masculino $(M=3.9, D P=0.1, n=46$ e $M=3.5, D P=0.1, n=47$, respetivamente). Verificou-se, ainda, uma diferença estatisticamente significativa no autocuidado, $F(1,88)$ $=5.31, p=.024, \eta^{2} p=.06$, com os participantes do sexo feminino $(M=5.1, D P=0.2, n=46)$ a apresentarem uma maior responsividade/responsabilidade corporal que os participantes do sexo masculino $(M=4.6, D P$ $=0.2, n=47$ ).

Finalmente, não foram encontrados efeitos principais da covariável escolaridade nas dimensões da apreciação da funcionalidade corporal, $F(1,88)=0.19, p=.663, \eta^{2} p=.00$ e do autocuidado, $F(1,88)=3.39$, $p=.069, \eta^{2} p=.04$, Por sua vez, a escolaridade exerceu efeitos nas dimensões da apreciação corporal, $F(1$, $88)=3.84, p=.053, \eta^{2} p=.04$, e da aceitação do corpo pelos outros, $F(1,88)=9.14, p=.003, \eta^{2} p=.09$, revelando que a escolaridade explica $4 \%$ da variância da apreciação corporal e $9 \%$ da avaliação da aceitação do corpo pelos outros (c.f. Tabela 2).

Tabela 2. Estatísticas descritivas das dimensões da ICP e análise de covariância com o grupo e o sexo como fatores e a escolaridade como covariável

\begin{tabular}{|c|c|c|c|c|c|c|c|c|}
\hline \multirow[t]{3}{*}{ Variável } & \multicolumn{2}{|c|}{ GC $(n=46)$} & \multicolumn{2}{|c|}{ GNC $(n=49)$} & \multicolumn{4}{|c|}{ Efeitos grupos*sexo } \\
\hline & $\begin{array}{c}\text { Sexo F } \\
(n=23)\end{array}$ & $\begin{array}{l}\text { Sexo M } \\
(n=23)\end{array}$ & $\begin{array}{c}\text { Sexo F } \\
(n=25)\end{array}$ & $\begin{array}{l}\text { Sexo M } \\
(n=24)\end{array}$ & & & & \\
\hline & $\mathrm{M} \pm \mathrm{DP}$ & $\mathrm{M} \pm \mathrm{DP}$ & $\mathrm{M} \pm \mathrm{DP}$ & $\mathrm{M} \pm \mathrm{DP}$ & $F$ & $g l$ & $p$ & $\eta^{2} p$ \\
\hline BAS-2 & $3.87 \pm 0.67$ & $3.57 \pm 0.81$ & $3.81 \pm 0.45$ & $3.87 \pm 0.67$ & 1.79 & $(1,88)$ & .184 & .02 \\
\hline BAOS & $3.96 \pm 0.75$ & $3.67 \pm 0.68$ & $3.93 \pm 0.60$ & $3.35 \pm 0.90$ & 1.55 & $(1,88)$ & .217 & .02 \\
\hline FAS & $3.98 \pm 0.59$ & $3.78 \pm 0.87$ & $4.30 \pm 0.48$ & $4.24 \pm 0.53$ & 0.45 & $(1,88)$ & .503 & .01 \\
\hline BRS & $5.01 \pm 0.92$ & $4.47 \pm 1.01$ & $5.03 \pm 1.05$ & $4.67 \pm 1.19$ & 0.17 & $(1,88)$ & .678 & .00 \\
\hline \multirow[t]{2}{*}{ Variáveis } & \multicolumn{2}{|c|}{ GC $(n=46)$} & \multicolumn{2}{|c|}{ GNC $(n=49)$} & \multicolumn{4}{|c|}{ Efeitos do grupo } \\
\hline & \multicolumn{2}{|c|}{$\mathrm{M} \pm \mathrm{DP}$} & \multicolumn{2}{|c|}{$\mathrm{M} \pm \mathrm{DP}$} & $F$ & $g l$ & $p$ & $\eta^{2} p$ \\
\hline BAS-2 & \multicolumn{2}{|c|}{$3.72 \pm 0.75$} & \multicolumn{2}{|c|}{$3.84 \pm 0.56$} & 2.41 & $(1,88)$ & .124 & .03 \\
\hline BAOS & \multicolumn{2}{|c|}{$3.82 \pm 0.72$} & \multicolumn{2}{|c|}{$3.64 \pm 0.81$} & 0.06 & $(1,88)$ & .801 & .00 \\
\hline FAS & \multicolumn{2}{|c|}{$3.88 \pm 0.74$} & \multicolumn{2}{|c|}{$4.27 \pm 0.50$} & 5.44 & $(1,88)$ & .022 & .06 \\
\hline BRS & \multicolumn{2}{|c|}{$4.74 \pm 0.99$} & \multicolumn{2}{|c|}{$4.86 \pm 1.13$} & 1.30 & $(1,88)$ & .258 & .02 \\
\hline \multirow[t]{2}{*}{ Variáveis } & \multicolumn{2}{|c|}{ Sexo F $(n=48)$} & \multicolumn{2}{|c|}{ Sexo $M(n=47)$} & \multicolumn{4}{|c|}{ Efeitos do sexo } \\
\hline & \multicolumn{2}{|c|}{$\mathbf{M} \pm \mathrm{DP}$} & \multicolumn{2}{|c|}{$\mathrm{M} \pm \mathrm{DP}$} & $F$ & $g I$ & $p$ & $\eta^{2} p$ \\
\hline BAS-2 & \multicolumn{2}{|c|}{$3.84 \pm 0.56$} & \multicolumn{2}{|c|}{$3.72 \pm 0.75$} & 1.30 & $(1,88)$ & .257 & .02 \\
\hline BAOS & \multicolumn{2}{|c|}{$3.94 \pm 0.67$} & \multicolumn{2}{|c|}{$3.51 \pm 0.81$} & 9.19 & $(1,88)$ & .003 & .10 \\
\hline FAS & \multicolumn{2}{|c|}{$4.15 \pm 0.56$} & \multicolumn{2}{|c|}{$4.02 \pm 0.74$} & 1.13 & $(1,88)$ & .290 & .01 \\
\hline BRS & \multicolumn{2}{|c|}{$5.02 \pm 0.98$} & \multicolumn{2}{|c|}{$4.57 \pm 1.10$} & 5.31 & $(1,88)$ & .024 & .06 \\
\hline Variáveis & & & & & Efei & da escol & aridade & \\
\hline & & & & & $F$ & $g I$ & $p$ & $\eta^{2} p$ \\
\hline BAS-2 & & & & & 3.84 & $(1,88)$ & .053 & .04 \\
\hline BAOS & & & & & 9.14 & $(1,88)$ & .003 & .09 \\
\hline FAS & & & & & 0.19 & $(1,88)$ & .663 & .00 \\
\hline BRS & & & & & 3.39 & $(1,88)$ & .069 & .04 \\
\hline
\end{tabular}

Nota. BAS-2 = apreciação corporal; BAOS = aceitação do corpo pelos outros; FAS = funcionalidade; BRS $=$ responsividade corporal; GC = grupo clínico; GNC = grupo não clínico. 


\section{A Relação Entre a Imagem Corporal Positiva e a Qualidade de Vida}

Para explorar como se associam as diversas variáveis da ICP, da QdV e da idade, foram realizadas correlações momento produto de Pearson, para o GC e não clínico (c.f. Tabela 3).

Grupo Clínico. Relativamente às dimensões da ICP, e tal como esperado, valores mais elevados de apreciação corporal (BAS-2) correspondem a valores igualmente mais elevados de aceitação corporal pelos outros (BAOS), $r(46)=.56, p \leq .001$, e de funcionalidade (FAS), $r(46)=.60, p \leq .001$. De referir, ainda, a presença de uma correlação moderada e estatisticamente significativa com o autocuidado corporal (BRS), $r(46)=.42, p=.003$, que por sua vez se correlaciona de forma positiva, moderada e significativa com a funcionalidade (FAS), $r(46)=.40, p=.006$ e com a aceitação do corpo por parte dos outros, $r(46)=.43, p$ $=.003$. Contudo, excecionalmente e ao contrário do que seria de esperar, a funcionalidade corporal não apresentou uma relação significativa com a aceitação do corpo por parte dos outros.

Por seu lado, a apreciação corporal (BAS-2) está, também, correlacionada de forma positiva e significativa com a QdV psicológica, $r(46)=.68, p \leq .001$, física, $r(46)=.60, p \leq .001$, relacional, $r(46)=$ $.53, p \leq .001$, ambiental, $r(46)=.42, p=.004$, e com a QdV geral, $r(46)=.38, p=.010$. A funcionalidade (FAS) está mais associada à QdV física, $r(46)=.58, p \leq .001$, à $\mathrm{QdV}$ geral, $r(46)=.49, p=.001 \mathrm{e}$ à $\mathrm{QdV}$ psicológica, $r(46)=.40, p=.006$. 0 autocuidado (BRS) encontra-se correlacionado de forma moderada, positiva e significativa com a $\mathrm{QdV}$ psicológica, $r(46)=.58, p \leq .001$, e relacional, $r(46)=.40, p=.006$. Finalmente, a aceitação do corpo pelos outros (BAOS) revela uma associação positiva e moderada com a $\mathrm{QdV}$ relacional, $r(46)=.41, p=.005$, e com a QdV psicológica, $r(46)=.39, p=.008$.

Por último, verificámos que a idade se associou, somente, de forma significativa (e negativa) com a QdV física, $r(46)=.30, p=.046$.

Grupo Não Clínico. Relativamente às facetas da ICP, verificámos, de igual forma, que a apreciação corporal (BAS-2) se correlaciona de forma positiva, moderada e estatisticamente significativa com todas as outras dimensões da ICP; com aceitação corporal pelos outros (BAOS), $r$ (49) = .64, $p \leq .001$; com a funcionalidade (FAS), $r(49)=.62, p \leq .001$, e com o autocuidado, $r(49)=.40, p=.004$. Constatou-se, também, uma associação positiva entre a funcionalidade (FAS) e a aceitação corporal pelos outros (BAOS), $r(49)=.48, p$ $\leq .001$, e o autocuidado (BRS), $r(49)=.31, p=.033$, e entre a aceitação do corpo pelos outros (BAOS) e o autocuidado corporal (BRS), $r(49)=.43, p=.002$.

Ademais, a apreciação corporal (BAS-2) está, também, correlacionada de forma positiva e significativa com a QdV psicológica, $r(49)=.64, p \leq .001$, e relacional, $r(49)=.52, p \leq .001$. Por seu lado, a aceitação corporal pelos outros (BAOS) está correlacionada de forma positiva e significativa com a QdV psicológica, $r(49)=.40, p=.004$, e relacional, $r(49)=.44, p=.002$. A apreciação da funcionalidade está correlacionada de forma positiva, moderada e significativa com a QdV psicológica, $r(49)=.64, p \leq .001$, e com a QdV relacional, $r(49)=.42, p=.002$.

Relativamente à idade, esta apresenta uma correlação estatisticamente significativa, positiva e baixa com a apreciação corporal (BAS-2), $r(49)=.33, p=.023$, com a aceitação do corpo pelos outros (BAOS), $r$ $(49)=.35, p=.015$, com a QdV relacional, $r(49)=.29, p=.043$, e com a QdV ambiental, $r(49)=.34, p=$ .017 . 
Tabela 3. Correlações de Pearson entre as Dimensões da ICP, QdV e Idade, no GC e GNC

\begin{tabular}{|c|c|c|c|c|c|c|c|c|c|c|}
\hline \multicolumn{11}{|c|}{ GC } \\
\hline & BAS-2 & BAOS & FAS & BRS & QdV_G & QdV_F & QdV_P & QdV_R & QdV_A & Idade \\
\hline BAS-2 & - & & & & & & & & & \\
\hline BAOS & $.56^{* *}$ & - & & & & & & & & \\
\hline FAS & $.60^{* *}$ & .19 & - & & & & & & & \\
\hline BRS & $.42^{* *}$ & $.43^{* *}$ & $.40^{* *}$ & - & & & & & & \\
\hline QdV_G & $.38^{*}$ & .16 & $.49^{* *}$ & .29 & - & & & & & \\
\hline QdV_F & $.60^{* *}$ & .10 & $.58^{* *}$ & .18 & $.55^{* *}$ & - & & & & \\
\hline QdV_P & $.68^{* *}$ & $.39^{* *}$ & $.40^{* *}$ & $.58^{* *}$ & $.49^{* *}$ & $.54^{* *}$ & - & & & \\
\hline QdV_R & $.53^{* *}$ & $.41^{* *}$ & $.37^{*}$ & $.40^{* *}$ & $.46^{* *}$ & $.50 * *$ & $.57^{* *}$ & - & & \\
\hline QdV_A & $.42^{* *}$ & .28 & .12 & $.33^{* *}$ & $.35^{* *}$ & $.38^{*}$ & $.56^{* *}$ & $.39 * *$ & - & \\
\hline \multirow[t]{3}{*}{ Idade } & .14 & .12 & -.15 & .11 & -.14 & $-.30 *$ & -.04 & -.15 & .17 & - \\
\hline & & & & & GNC & & & & & \\
\hline & BAS-2 & BAOS & FAS & BRS & & QdV_F & QdV_P & QdV_R & QdV_A & Idade \\
\hline BAS-2 & - & & & & & & & & & \\
\hline BAOS & $.64^{* *}$ & - & & & & & & & & \\
\hline FAS & $.62^{* *}$ & $.48^{* *}$ & - & & & & & & & \\
\hline BRS & $.40^{* *}$ & $.43^{* *}$ & $.31^{*}$ & - & & & & & & \\
\hline QdV_F & $.30^{* *}$ & .08 & $.33^{*}$ & .05 & & - & & & & \\
\hline QdV_P & $.64^{* *}$ & $.40^{* *}$ & $.64^{* *}$ & $.33^{*}$ & & $.46^{* *}$ & - & & & \\
\hline QdV_R & $.52^{* *}$ & $.44^{* *}$ & $.42^{* *}$ & $.34^{*}$ & & $.41^{* *}$ & $.50 * *$ & - & & \\
\hline QdV_A & .26 & .08 & $.35^{*}$ & .13 & & $.55^{* *}$ & $.59^{* *}$ & $.40^{* *}$ & - & \\
\hline Idade & $.33^{*}$ & $.35^{*}$ & .12 & .09 & & .08 & .23 & $.29 *$ & $.34 *$ & - \\
\hline
\end{tabular}

\section{Modelo de Predição para a Qualidade de Vida}

Foi realizada uma regressão linear múltipla, método confirmatório (enter), para se explorar quais os principais preditores da qualidade de vida geral (QdV_G), independentemente do grupo de pertença. Desta forma, foram incluídas como variáveis independentes a apreciação corporal (BAS-2), a apreciação da funcionalidade corporal (FAS), a aceitação do corpo pelos outros (BAOS), a responsividade corporal/autocuidado (BRS) e o grupo (0 - grupo clínico e 1- grupo não clínico) ${ }^{6}$. Foi obtido um modelo estatisticamente significativo que explica cerca de $47 \%\left(R_{a j}^{2}=.47\right)$ da QdV geral, $F(5,89)=17.87, p \leq .001$. De todos os preditores, apenas a FAS, $t(5)=3.75, p \leq .001, I C=(5.21-16.92), \beta=.38, B=11.06, D P=2.95$, e o grupo, $t(5)=5.16, p \leq .001, I C=(9.75-21.95), \beta=.41, B=15.85, D P=3.07$, são preditores estatisticamente significativos, apresentando este último o maior poder explicativo. Desta forma, pode-se concluir que quase $50 \%$ da variância de uma boa qualidade de vida, nesta amostra de participantes, é explicada pela ausência de IRC e pela apreciação da funcionalidade corporal (c.f. Tabela 4).

Tabela 4. Modelo de Regressão Linear Múltipla Para a Qualidade de Vida Geral

\begin{tabular}{lcccccc}
\hline & $\boldsymbol{B}$ & $\boldsymbol{D P}$ & $\boldsymbol{\beta}$ & $\boldsymbol{t}$ & $\boldsymbol{p}$ & $\boldsymbol{I C ~ 9 5 \%}$ \\
\hline BAS-2 & 3.87 & 3.27 & .13 & 1.18 & .239 & {$[-2.62-10.36]$} \\
BAOS & -0.44 & 2.43 & -.02 & -0.18 & .857 & {$[-5.26-4.38]$} \\
FAZ & 11.06 & 2.94 & .38 & 3.75 & $\mathbf{. 0 0 0}$ & {$[5.21-16.92]$} \\
BRS & 0.48 & 1.56 & .03 & 0.31 & .761 & {$[-2.63-3.58]$} \\
Grupo $^{\mathbf{a}}$ & 15.85 & 3.07 & .41 & 5.16 & $\mathbf{. 0 0 0}$ & {$[9.75-21.95]$} \\
\hline
\end{tabular}

\footnotetext{
${ }^{6}$ De referir que a variável "idade" não foi incluída no modelo, tal como proposto na H3.1, uma vez que esta não apresentou uma correlação estatisticamente significativa com a QdV geral.
} 
Nota. BAS-2 = apreciação corporal; BAOS = aceitação do corpo pelos outros; FAS = funcionalidade; BRS = responsividade/autocuidado corporal; a 0 - grupo clínico e 1 - grupo não clínico.

\section{DISCUSSÃo}

O presente trabalho teve como principal objetivo estudar a ICP num grupo de pessoas com IRC, em processo de hemodiálise, explorando-se o seu impacto na qualidade de vida. Para tal foram realizadas diferentes análises estatísticas de forma a caraterizar, avaliar e descrever a relação entre um conjunto de variáveis associadas à ICP e à QdV em indivíduos com IRC, assim como comparar este grupo com um grupo de pessoas sem doença crónica.

Ao contrário do que seria de esperar (H1), a apreciação corporal, a aceitação do corpo pelos outros e o autocuidado não apresentaram diferenças em função do grupo, verificando-se níveis similares de ICP (acima da média escalar). Tal poder-se-á dever à vertente subjetiva da apreciação corporal, à sua abertura à mudança por influência social ou outras fontes (reciprocidade) e ao próprio conceito de apreciação corporal. Efetivamente, mesmo com limitações físicas, pode haver uma ICP, uma vez que esta não simboliza ter um corpo perfeito alinhado com o ideal cultural, mas antes a capacidade para apreciar a sua beleza única, aceitar e admirar o corpo como ele é, sentir-se confortável e feliz, enfatizando os seus aspetos positivos, internalizando as informações positivas sobre o mesmo e rejeitando/reformulando as negativas. Assim, assume-se como um fator potenciador da apreciação corporal em pessoas com alterações visíveis na aparência ou deficiência física, a aceitação do corpo e a concentração nos seus aspetos positivos (Grogan, 1999; Tylka \& Wood Barcalow, 2015a; Wood-Barcalow et al., 2010).

A ausência de diferenças entre grupos encontrada na BAOS, por seu lado, poder-se-á relacionar com o facto de ambos os grupos de participantes apresentarem uma boa rede de suporte social (familiar e de amizades). Efetivamente, o suporte social tem vindo a ser considerado como uma componente essencial para o desenvolvimento e manutenção de uma ICP (Bailey et al., 2015). Assim, pessoas com relações mais apoiantes serão mais capazes de filtrar eventuais opiniões/comentários menos positivos, ao mesmo tempo que terão uma maior capacidade de se aceitarem por aquilo que são e fazem.

De referir, ainda, que foram poucas as alterações corporais referidas pela amostra clínica na variável “Que alterações corporais surgiram como consequência da doença e/ou tratamentos?", pelo que a ausência de diferenças se poderá justificar pelo facto de as mudanças corporais não serem assim tão visíveis ou percecionadas como relevantes para estes participantes. Concomitantemente, o presente instrumento avalia as alterações corporais em termos de forma e peso corporal pelo que, mesmo quando são referidas diferenças em termos de peso pelos participantes, estas podem ser interpretadas como positivas. Tal aspeto reforça o conceito de ICP como um construto mais abrangente que a mera valorização da aparência ou da satisfação com a forma e peso corporal (muito associadas a medidas de avaliação da IC). Assim, uma pessoa pode valorizar mais outras dimensões do seu corpo (e.g., apreciação da funcionalidade corporal) para além do peso e, por isso, relativizar a sua importância. Relativamente ao autocuidado, Tsay (2003) revela que pacientes em hemodiálise apresentam um baixo nível de autoeficácia no autocuidado. Referindo que, quanto menor a autoeficácia no autocuidado de um paciente, menor a confiança que tem na sua capacidade para o autocuidado, tornando-se menos disposto a executar ações necessárias para tal. Ora, estando os hemodialisados mais restritos em termos de ingestão alimentar e hídrica, por exemplo, seria de esperar que esta dimensão fosse estatisticamente inferior ao grupo saudável, tal como referido por Tsay (2003). Contudo, tais diferenças não se verificaram. Pacientes em hemodiálise são submetidos a tratamento dialítico, que é um processo de longo prazo. Por esse motivo, eles são obrigados a gerir a sua doença, adotando estratégias de autocuidado para sobreviver (Richard \& Engebretson, 2010). Adicionalmente, o facto de terem passado por um momento de crise (confrontação com o diagnóstico e tratamento) fez com que estes sujeitos, possivelmente, começassem a atribuir maior importância à apreciação do seu estado de saúde, não o tomando como garantido, ouvindo e cuidando do corpo, estando em sintonia com ele.

De todas as dimensões da ICP avaliadas, apenas a apreciação da funcionalidade corporal apresentou diferenças estatisticamente significativas em função do grupo, sendo mais elevada no GNC (como esperado), apesar de ambos os grupos apresentarem valores superiores à média da escala. Sabendo que a funcionalidade do corpo é definida como tudo o que o corpo pode fazer ou é capaz de fazer, valorizando-o, respeitando-o e honrando-o por isso, englobando funções relacionadas com as capacidades físicas (e.g., ter flexibilidade, conseguir andar) ou os processos internos (e.g., digerir alimentos, curar de um resfriado), será natural que pessoas com doença renal percecionem níveis mais reduzidos de funcionalidade e das capacidades inerentes ao seu corpo, justificado pelas diversas limitações físicas que a IRC acarreta (Alleva et al., 2017; Bailey et al., 2015).

Relativamente à $\mathrm{H} 2$, e tal como esperado, não foram encontradas diferenças entre homens e mulheres quanto à apreciação corporal e à apreciação da funcionalidade corporal, independentemente do grupo, indo de encontro a alguns estudos neste âmbito (Meneses et al., 2019; Razmus \& Razmus, 2017) e, 
paralelamente, o sexo feminino apresentou valores mais elevados de responsividade corporal que o sexo masculino, tal como hipotetizado. Contudo as mulheres revelaram níveis significativamente mais elevados que os homens na perceção da aceitação do corpo pelos outros. Assim, o sexo feminino parece apresentar uma interpretação da avaliação do seu corpo pelos amigos, pela família e pelo par amoroso como mais positiva, comparativamente ao sexo masculino. Tal aspeto poder-se-á relacionar com a presença de uma rede de apoio importante e que manifesta de forma direta ou indireta essa aceitação pelo corpo do outro. De referir que as mulheres recebem mais vezes essa valorização relativamente ao seu corpo do que os homens, uma vez que é culturalmente mais aceite dar-se esse feedback relativamente ao corpo das mulheres do que aos homens, que, por norma, são mais orientados para a atividade (activity oriented) e menos para a partilha dialógica (Jones \& Crawford, 2006).

Relativamente aos níveis mais elevados de responsividade corporal ou autocuidado no sexo feminino, sabe-se que o autocuidado se refere ao investimento no cuidado com o corpo e nas suas necessidades físicas (Tylka, 2011); manifestando-se, por exemplo, numa maior proteção solar, procura de atenção médica e negativamente relacionado com comportamentos de consumo de álcool e cigarros (Wood-Barcalow et al., 2010). Ora, possivelmente devido a pressões culturais e educacionais (e.g., papéis tradicionais de género) este tipo de comportamentos de autocuidado e responsabilidade corporal é, ainda, menos frequente nos homens (Pengpid \& Peltzer, 2015), o que pode estar a justificar a presença de diferenças neste domínio.

Relativamente à hipótese 3, verificou-se uma associação positiva e significativa entre as dimensões da ICP apenas no GNC. Contudo, no GC, apesar da apreciação corporal e o autocuidado se associarem com todas as dimensões da ICP, não se verificou uma associação significativa entre a apreciação da funcionalidade corporal e a aceitação do corpo pelos outros. Assim, no GC a aceitação da funcionalidade parece ser mais independente da apreciação por parte dos outros dessa funcionalidade do que no grupo de pessoas sem IRC, cuja apreciação da funcionalidade corporal estará associada à aceitação corporal por parte dos outros. Tal poderá justificar-se pelo facto da apreciação da funcionalidade corporal e as questões de capacidade corporal, no GC, estarem mais centradas na vertente individual, ou seja, com as preocupações de saúde, e menos com a aceitação por parte dos outros dessa funcionalidade.

Relativamente à relação entre a ICP e a QdV, a BAS relacionou-se com todas as dimensões da QdV em ambos os grupos, como seria de esperar, à exceção da QdV ambiental, no GNC. Ou seja, a QdV ambiental parece não ter qualquer tipo de impacto na apreciação do corpo, e vice-versa, somente no GNC. Tal aspeto poder-se-á dever ao facto da QdV ambiental facilitar um conjunto de vivências sociais, acessos a bens e serviços que contribuem para uma maior inclusão de pessoas com IRC, o que se manifestará em níveis superiores de valorização e apreciação corporal.

0 mesmo acontece com a apreciação da funcionalidade (FAS) que se correlaciona com todas as dimensões da QdV, como seria de esperar, com exceção da QdV ambiental no GC. Este resultado poderá ser explicado pelo facto da apreciação da funcionalidade corporal no GC não estar tão dependente de condições/aspetos ambientais, mas de aspetos mais psicológicos. Apesar de poderem ter determinadas condições ambientais satisfeitas, a apreciação da funcionalidade em pessoas com IRC exige estratégias de coping mais internas para tentar lidar com todos os desafios inerentes a uma maior perda de funcionalidade.

A BAOS e a BRS, por sua vez, só se relacionam com a QdV psicológica e relacional, em ambos os grupos (e, no caso da BRS, também com a QdV ambiental no GC). Assim, a QdV psicológica e relacional parece apresentar uma relação mais consistente com o autocuidado e a perceção do corpo pelos outros. De facto, vivências sociais significativas e proximais prezam o autocuidado e a valorização de si e do seu corpo. Por seu lado, um maior investimento no autocuidado e uma perceção positiva de si e do seu corpo, estão associados a níveis mais elevados de bem-estar psicológico e ao cuidado das relações sociais encetadas.

Relativamente à H3.1., seria expectável uma associação positiva entre a idade e a ICP e a QdV. Esta hipótese foi parcialmente confirmada. No GNC, a idade correlaciona-se de forma estatisticamente significativa com a apreciação corporal e a aceitação corporal pelos outros. Isto é, à medida que a idade aumenta, maior é a aceitação corporal do próprio e perceção da aceitação corporal pelos outros, pois, tal como sugerem Esnaola e colaboradores (2010), as pessoas atribuem menos importância à sua aparência física, à medida que se aproximam da idade adulta média e tardia, e valorizam outras dimensões do seu corpo e da sua corporalidade. Tal aspeto pode ser protetor face às autoavaliações negativas que podem manter-se associadas à imagem corporal e à pressão para uma determinada aparência e corpo jovens.

Contudo, ao contrário do que seria de esperar, a apreciação da funcionalidade não aumenta à medida que a idade aumenta. Tal aspeto poder-se-á dever ao facto da presente amostra ser constituída principalmente por indivíduos com idades mais avançadas e, por esse motivo, levantou-se a hipótese de a relação entre a idade e a funcionalidade não ser linear, mas antes curvilínea, em U invertido. Depois de realizada uma regressão quadrática, verifica-se que a apreciação da funcionalidade corporal aumenta até 
aos 50 anos de idade, aproximadamente, mantendo-se estável mais ou menos até aos 55 anos. A partir dessa idade, verificou-se um declínio da apreciação da funcionalidade, mais acentuado a partir dos 70 anos. Assim, conclui-se que a presente relação é melhor explicada por uma relação curvilínea (função quadrática), $F(2,92)=3.12, p=.049, r^{2}=.063$, do que por uma relação linear (função linear), $F(1,93)=$ $1.23, p=.270, r^{2}=.013$ nesta amostra em particular, que abrange uma população com idade mais avançada e, portanto, com maior probabilidade de ter declínios mais acentuados ao nível da funcionalidade, comparativamente a idades mais jovens. Desta forma, será fundamental o papel da promoção da melhoria da apreciação da funcionalidade corporal e do seu papel, particularmente a partir de uma certa idade.

No GNC, encontrou-se, ainda, uma associação positiva entre a idade e a QdV relacional e ambiental. Tal aspeto poder-se-á dever a um aumento da estabilidade financeira, investimento no lar (QdV ambiental), o aumento da seletividade e otimização das relações significativas e mais tempo para o lazer (QdV relacional), fortemente associado à idade da reforma, à saída dos filhos de casa dos pais e a uma ressignificação das palavras lar e vida.

Por sua vez, no GC, a idade não se correlaciona com nenhuma das dimensões da ICP e apenas com a QdV física (de forma negativa). Assim, há medida que a idade aumenta, a qualidade de vida física diminui nestes participantes, o que seria expectável, pois para além de um declínio natural das capacidades corporais com a idade, o facto de terem uma doença crónica com as implicações físicas e psicológicas que acarreta, torna esta perceção de qualidade de vida no domínio físico mais acentuada no grupo em questão.

Finalmente, relativamente à hipótese 4, de todas as facetas da ICP incluídas no modelo (a apreciação corporal, a apreciação da funcionalidade e a responsividade corporal ou autocuidado), apenas a apreciação da funcionalidade corporal e o grupo se revelaram preditores estatisticamente significativos da QdV geral. $\mathrm{Na}$ verdade, ter ou não uma doença crónica, neste caso, ter IRC e fazer hemodiálise, ou não ter esta condicionante clínica, parece ser o fator que mais influencia significativamente a QdV geral, o que é consonante com outros estudos que associam negativamente a QdV a condições clínicas crónicas (Megari, 2013).

Paralelamente, é a vertente mais funcional da ICP que apresenta um impacto maior na QdV geral. Indaga-se sobre o porquê de as outras dimensões não apresentarem este poder preditivo, particularmente a apreciação corporal, associada à QdV geral (no GC). Hipotetiza-se que, apesar da aceitação e valorização corporal poder ser uma dimensão relevante, quando colocada conjuntamente com a apreciação da funcionalidade, perde a sua força na explicação da QdV. Tal poderá acontecer pelo facto de as pessoas valorizarem mais a vertente daquilo que o corpo ainda é capaz de fazer, principalmente os participantes mais velhos. Tiggemann (2004) argumenta que, embora a aparência e aceitação corporais sejam importantes à medida que se envelhece, é a satisfação com os aspetos funcionais do corpo que prevalece. Ora, de referir que a presente amostra é constituída, essencialmente, por população adulta com uma média de idades de 55 anos, no GNC, ou 60 anos, no GC. Tais características amostrais podem justificar o porquê de apenas a FAS apresentar esta capacidade preditiva sobre a QdV geral, uma vez que a incapacidade funcional aumenta com a idade (Greenglass et al., 2007). Paralelamente, dever-se-á realçar o facto de a funcionalidade ser uma das dimensões com mais impacto na mudança da ICP geral (Alleva et al., 2018) e, assim sendo, faz sentido que ela seja também a dimensão com mais potencial e capacidade de alterar a QdV.

\section{CONCLUSÃO}

O presente estudo pretendeu explorar a relação da ICP em pessoas com IRC, em processo de hemodiálise, e pessoas sem IRC, e de que forma esta se associa à QdV. Relativamente à $\mathrm{H} 1$ e H2, foram encontradas diferenças quanto ao sexo nas dimensões da aceitação do corpo pelos outros e do autocuidado, superiores no sexo feminino; bem como diferenças quanto ao grupo de pertença (GC ou GNC), na apreciação da funcionalidade corporal, superior no GNC.

No que concerne à H3 e H3.1., de uma forma geral, verificou-se que a apreciação corporal, a perceção de funcionalidade corporal, a perceção da aceitação corporal por parte dos outros e o autocuidado, se associam com os diferentes domínios da QdV, com associações significativas tanto em pessoas com IRC como nas que não manifestam nenhuma doença crónica. De referir, ainda, a relação positiva da idade com a apreciação corporal, a aceitação corporal pelos outros, a QdV relacional e ambiental, no GNC, bem como uma relação negativa da idade com a QdV física nos participantes com IRC.

Por último (H4), destaca-se o papel desempenhado pelo grupo de pertença (GC ou GNC) e pela apreciação da funcionalidade corporal como importantes preditores da qualidade de vida, apresentando este último maior poder explicativo.

Apesar da relevância dos resultados, é importante referir as limitações que acompanham este estudo. A primeira grande limitação circunscreve-se ao domínio da recolha da amostra, feita por duas pessoas e por dois meios diferentes (online e presencial). A presença de dois investigadores pode induzir 
diferentes resultados, devido às diferentes posturas que assumem. Verifica-se o mesmo com dois tipos de recolha, que podem suscitar essas mesmas diferenças. De referir a preferência pelo método presencial, embora possa induzir algum viés, fruto do contacto e de preferências naturais. Apesar da recolha online conferir uma maior amplitude de recolha, não permite o controlo do preenchimento do questionário, nomeadamente dos dados sociodemográficos e clínicos dos participantes. De referir, ainda, que o questionário foi administrado presencialmente durante o processo de hemodiálise, em condições de pouca mobilidade física, o que obrigou a que o mesmo fosse lido e preenchido pelas investigadoras o que suscita ainda maior viés e maior desejabilidade social.

Relativamente ao questionário em si, este revelou-se como bastante extenso, o que dificultou o recrutamento de amostra, o término do mesmo e a manutenção da atenção e motivação.

No que concerne à amostra, o facto de se ter recorrido a uma amostragem não-probabilística inviabiliza a generalização para a população e a pouca representatividade da mesma. No entanto, seria importante tentar-se alargar e diversificar mais a amostra, em termos de zonas geográficas e de escolaridade, o que se tentou fazer com a recolha realizada num centro hospitalar (público) do grande Porto e outra realizada num centro de diálise (privado) de uma zona do interior. Para além disso, o facto da amostra ser maioritariamente envelhecida, exigia, em muitos casos, um teste de realidade, feito por um significativo ou pessoa da área hospitalar com conhecimento da história médica. Finalmente, as circunstâncias de pandemia, devido à COVID-19, limitaram a recolha de dados e, portanto, o condicionamento do número de participantes da amostra clínica.

Em termos metodológicos, seria interessante futuramente recorrer a uma metodologia qualitativa, tendo por base os discursos dos participantes, tentando-se aceder aos significados da imagem corporal positiva, à forma como falam do seu corpo e vivência corporal na sua situação clínica, e às implicações na sua qualidade de vida, com o objetivo de enriquecer os dados e as interpretações obtidas. Seria relevante, ainda, tentar avaliar os participantes que recorrem ao transplante renal, antes e depois dessa intervenção, numa tentativa de se perceber eventuais mudanças ao nível da imagem corporal e da qualidade de vida e aceder às perceções dos mais significativos relativamente às dimensões avaliadas, valorizando-se uma abordagem mais sistémica dos dados.

Salienta-se, de qualquer forma, o caráter inovador deste estudo, numa área de investigação ainda bastante recente (a da imagem corporal positiva) e ausente em termos de investigação nesta população clínica em particular, mas em populações clínicas de uma forma geral. Paralelamente, apresenta implicações importantes para a prática clínica. Na verdade, a realização deste estudo colocou a tónica nas inúmeras vantagens de se intervir na ICP, essencialmente pelas implicações positivas que produz ao nível dos diversos domínios da qualidade de vida, independentemente de se ter, ou não, doença crónica.

Uma boa forma de promover a QdV geral poderá ser através do desenvolvimento da apreciação da funcionalidade corporal uma vez que esta atua como um forte propulsor desta dimensão, nesta amostra clínica em particular. Alia-se, ainda, a crescente preocupação quanto às consequências de um grande número de idosos com limitações na capacidade funcional, devido ao declínio funcional associado ao aumento da idade, o que torna ainda mais premente a intervenção nesta questão (Greenglass et al., 2007). 0 papel da funcionalidade, da apreciação corporal, da aceitação corporal por parte dos significativos e do autocuidado reportam para a necessidade de se desenvolverem intervenções integrativas junto destes doentes, abrangendo os domínios físicos e emocionais, garantindo uma adaptação às limitações psicossociais e promovendo uma melhoria na QdV desta população. Ora, a par de muitos outros estudos (e.g., Alleva et al., 2018), seria deveras interessante realizar intervenções onde o corpo aparece como objeto central. Tal aspeto é passível de ser desenvolvido através do foco na funcionalidade de uma forma holística, nomeadamente, pelo foco nas capacidades físicas e sensações corporais. Outras intervenções, que não são necessariamente explícitas ou exclusivamente focadas na funcionalidade do corpo, também podem promover uma orientação baseada na funcionalidade em relação ao corpo. Destacam-se as atividades físicas e intervenções baseadas no ioga, bem como algumas formas de dança, contribuindo para uma melhoria da imagem corporal positiva, ajudando os indivíduos a desviar sua atenção para os aspetos funcionais do corpo (McLean et al., 2011).

Em suma, espera-se que este estudo seja propulsor de futura investigação com esta população, apelando-se a uma continuidade da curiosidade científica nesta área e no investimento em mudanças na prática clínica, visando-se a promoção da qualidade de vida destas pessoas, nomeadamente através do desenvolvimento de uma ICP.

\section{REFERÊNCIAS}

Alleva, J., Diedrichs, P., Halliwell, E., Martijn, C., Stuijfzand, B., Treneman-Evans, G., \& Rumsey, N. (2018). A randomised-controlled trial investigating potential underlying mechanisms of a functionality-based 
approach to improving women's body image. Body Image, 25, 85-96. https://doi.org/10.1016/j.bodyim.2018.02.009

Alleva, J., Tylka, T., \& Van Diest, A. (2017). The Functionality Appreciation Scale (FAS): Development and psychometric evaluation in U.S. community women and men. Body Image, 23, 28-44. https://doi.org/10.1016/j.bodyim.2017.07.008

Atari, M. (2016). Factor structure and psychometric properties of the Body Appreciation Scale-2 in Iran. Body Image, 18, 1-4. https://doi.org/10.1016/j.bodyim.2016.04.006

Avalos, L., \& Tylka, T. (2006). Exploring a model of intuitive eating with college women. Journal of Counseling Psychology, 53(4), 486-497. https://doi.org/10.1037/0022-0167.53.4.486

Bailey, K., Cline, L., \& Gammage, K. (2016). Exploring the complexities of body image experiences in middle age and older adult women within an exercise context: The simultaneous existence of negative and positive body images. Body Image, 17, 88-99. https://doi.org/10.1016/j.bodyim.2016.02.007

Bailey, K., Gammage, K., Ingen, C., \& Ditor, D. (2015). "It's all about acceptance": A qualitative study exploring a model of positive body image for people with spinal cord injury. Body Image, 15, 24-34. https://doi.org/10.1016/j.bodyim.2015.04.010

Barbosa, M., Vieira, F., Brandão, M., Meneses, L., Fernandes, R., \& Torres, S. (2018, june 12-14). Measurement of positive body image's facets: Psychometric properties of the BESAA and BAOS in Portuguese adolescents and adults [Poster session]. 8th biennial Appearance Matters Conference, Bath, United Kingdom.

Daubenmier, J.J. (2005). The relationship of yoga, body awareness, and body responsiveness to selfobjetification and disorderer eating. Psychology of Women Quarterly, 29(2), 207-219. https://doi.org/10.1111/j.1471-6402.2005.00183.x

Esnaola, I., Rodríguez-Fernández, A., \& Goñi, A. (2010). Body dissatisfaction and perceived sociocultural pressures: gender and age differences. Insatisfacción corporal y presión sociocultural percibida: diferencias asociadas al sexo y a la edad. Salud Mental, 33(1), 21-29. https://www.redalyc.org/articulo.oa?id=582/58215621003

Finnegan-John, J., \& Thomas, V. (2012). The psychosocial experience of patients with end-stage renal disease and its impact on quality of life: Findings from a needs assessment to shape a service. ISRN Nephrology, 2013, 308986. https://doi.org/10.5402/2013/308986

Greenglass, E., Fiksenbaum, L., \& Eaton, J. (2007). The relationship between coping, social support, functional disability and depression in the elderly. Anxiety, Stress, and Coping, 19(1), 15-31. https://doi.org/10.1080/14659890500436430

Grogan, S. (1999). Body image: Understanding body dissatisfaction in men, women and children. Routledge.

Jovic, M., Sforza, M., Jovanovic, M., \& Jovic, M. (2016). The acceptance of cosmetic surgery scale: Confirmatory factor analyses and validation among Serbian adults. Current Psychology, 36, 707-718. https://doi.org/10.1007/s12144-016-9458-7

Jones, D., \& Crawford, J. (2006). The peer appearance culture during adolescence: Gender and body mass variations. Journal of Youth and Adolescence, 35(243), 257-269. https://doi.org/10.1007/s10964005-9006-5

Kline, R.B. (2011). Principles and practice of structural equation modelling (3 $3^{\text {rd }}$ ed.). Guilford Press.

Lemoine, J., Konradsen, H., Jensene, A., Roland-Lévy, C., Nyg, P., Khalaf, A., \& Torres, S. (2018). Factor structure and psychometric properties of the Body Appreciation Scale-2 among adolescents and young adults in Danish, Portuguese, and Swedish. Body Image, 26, 1-9. https://doi.org/10.1016/j.bodyim.2018.04.004

Liew, G., Mitchell, P., Wong, T., \& Wang, J. (2012). Retinal microvascular signs are associated with chronic kidney disease in persons with and without diabetes. Kidney Blood Press Res, 35, 589-594. https://doi.org/10.1159/000339173

McLean, S., Paxton, S., \& Wertheim, E. (2011). A body image and disordered eating intervention for women in midlife: A randomized controlled trial. Journal of Consulting and Clinical Psychology, 79(6), 751758. https://doi.org/10.1037/a0026094

Megari, K. (2013). Quality of Life in Chronic Disease Patients. Health psychology research, 1(3), e27. https://doi.org/10.4081/hpr.2013.e27

Meneses, L., Torres, S., Miller, K., \& Barbosa, M. (2019). Extending the use of the Body Appreciation Scale 2 in older adults: A Portuguese validation study. Body Image, 29, 74-81. https://doi.org/10.1016/j.bodyim.2019.02.011

Moreira, H., \& Canavarro, M. (2010). A longitudinal study about the body image and psychosocial adjustment of breast cancer patients during the course of the disease. European Journal of Oncology Nursing, 14(4), 263-270. https://doi.org/10.1016/j.ejon.2010.04.001 
Oldenburg, C., Boll, D., Nicolaije, K., Vos, M., Pijnenborg, J., Coebergh, J., Beijer, S., Poll-Franse, L., \& Ezendam, N. (2013). The relationship of body mass index with quality of life among endometrial cancer survivors: A study from the population-based PROFILES registry. Gynecologic Oncology, 129, 216221. https://doi.org/10.1016/j.ygyno.2012.12.041

Partridge, K., \& Robertson, N. (2011). Body-image disturbance in adult dialysis patients. Disability and Rehabilitation, 33(6), 504-510. https://doi.org/10.3109/09638288.2010.498556

Pengpid, S., \& Peltzer, K. (2015). Gender differences in health risk behaviour among university students: An international study. Gender and Behaviour, 13(1), 6576-6583. http://hdl.handle.net/20.500.11910/1651

Razmus, M., \& Razmus, W. (2017). Evaluating the psychometric properties of the Polish version of the Body Appreciation Scale-2. Body Image, 23, 45-49. https://doi.org/10.1016/j.bodyim.2017.07.004

Richard, C., \& Engebretson, J. (2010). Negotiating living with an anteriovenous fistula for hemodialysis. Nephrology Nursing Journal, 37(4), 363-375.

Serra, A., Canavarro, M., Simões, M., Pereira, M., Gameiro, S., Quartilho, M., Rijo, D., Corona, C., \& Paredes, T. (2006). Estudos psicométricos do instrumento avaliação da Qualidade de Vida da Organização Mundial de Saúde (WHOQOL-Bref) para Português de Portugal. Psiquiatria Clínica, 27(1), 41-49.

Swami, V., \& Ng, S. (2015). Factor structure and psychometric properties of the Body Appreciation Scale-2 in university students in Hong Kong. Body Image, 15, 68-71. https://doi.org/10.1016/j.bodyim.2015.06.004

Tiggemann, M. (2004). Body image across the adult life span: Stability and change. Body Image, 1(1), 2941. https://doi.org/10.1016/S1740-1445(03)00002-0

Tsay, S. (2003). Self-efficacy training for patients with end-stage renal disease. Issues and innovations in nursing practice, 43(4), 370-375. https://doi.org/10.1046/j.1365- 2648.2003.02725.x

Tylka, T. L. (2011). Positive psychology perspectives on body image. In T. F. Cash \& L. Smolak (Eds.), Body image: A handbook of science, practice, and prevention (2nd ed., pp. 56-64). Guilford Press.

Tylka, T., \& Wood-Barcalow, N. (2015a). What is and what is not positive body image? Conceptual foundations and construct definition. Body Image, 14, 118-129. https://doi.org/10.1016/j.bodyim.2015.04.001

Tylka, T., \& Wood-Barcalow, N. (2015b). The Body Appreciation Scale-2: Item refinement and psychometric evaluation. Body Image, 12, 53-67. https://doi.org/10.1016/j.bodyim.2014.09.006

WHOQOL (1998). The world health organization quality of life assessment (WHOQOL): Development and general psychometric properties. Social science \& medicine, 46(12), 1569-1585. https://doi.org/10.1016/s0277-9536(98)00009-4

Wood-Barcalow, N., Tylka, T., \& Augustus-Horvath, C. (2010). "But I like my body": Positive body image characteristics and a holistic model for young-adult women. Body Image, 7(2), 106-116. https://doi.org/10.1016/j.bodyim.2010.01.001 


\section{Historial do artigo}

Recebido

$11 / 01 / 2021$

Aceite

$26 / 05 / 2021$

Publicado

$20 / 12 / 2021$ 